\title{
MetaComp: comprehensive analysis software for comparative meta-omics including comparative metagenomics
}

\author{
Peng Zhai ${ }^{1}$, Longshu Yang ${ }^{1,2}$, Xiao Guo², Zhe Wang ${ }^{1,2}$, Jiangtao Guo ${ }^{1,2}$, Xiaoqi Wang ${ }^{1,2}$
} and Huaiqiu Zhu ${ }^{1,2,3^{*}}$

\begin{abstract}
Background: During the past decade, the development of high throughput nucleic sequencing and mass spectrometry analysis techniques have enabled the characterization of microbial communities through metagenomics, metatranscriptomics, metaproteomics and metabolomics data. To reveal the diversity of microbial communities and interactions between living conditions and microbes, it is necessary to introduce comparative analysis based upon integration of all four types of data mentioned above. Comparative meta-omics, especially comparative metageomics, has been established as a routine process to highlight the significant differences in taxon composition and functional gene abundance among microbiota samples. Meanwhile, biologists are increasingly concerning about the correlations between meta-omics features and environmental factors, which may further decipher the adaptation strategy of a microbial community.

Results: We developed a graphical comprehensive analysis software named MetaComp comprising a series of statistical analysis approaches with visualized results for metagenomics and other meta-omics data comparison. This software is capable to read files generated by a variety of upstream programs. After data loading, analyses such as multivariate statistics, hypothesis testing of two-sample, multi-sample as well as two-group sample and a novel function-regression analysis of environmental factors are offered. Here, regression analysis regards meta-omic features as independent variable and environmental factors as dependent variables. Moreover, MetaComp is capable to automatically choose an appropriate two-group sample test based upon the traits of input abundance profiles. We further evaluate the performance of its choice, and exhibit applications for metagenomics, metaproteomics and metabolomics samples.
\end{abstract}

Conclusion: MetaComp, an integrative software capable for applying to all meta-omics data, originally distills the influence of living environment on microbial community by regression analysis. Moreover, since the automatically chosen two-group sample test is verified to be outperformed, MetaComp is friendly to users without adequate statistical training. These improvements are aiming to overcome the new challenges under big data era for all meta-omics data. MetaComp is available at: http://cqb.pku.edu.cn/ZhuLab/MetaComp/ and https://github.com/ pzhaipku/MetaComp/.

Keywords: Comparative metagenomics, Comparative meta-omics, Statistical analysis, Visualization, Graphical user interface

\footnotetext{
*Correspondence: hqzhu@pku.edu.cn

'State Key Laboratory for Turbulence and Complex Systems, Department of

Biomedical Engineering, College of Engineering, Peking University, 100871

Beijing, China

${ }^{2}$ Center for Quantitative Biology, Peking University, 100871 Beijing, China

Full list of author information is available at the end of the article
} 


\section{Background}

High-throughput meta-omic approaches over the last few years have facilitated researches on understanding of the unculturable majority of microorganisms on earth. Environmental and clinical microbiota samples are characterized in metagenomics, metatranscriptomics, metaproteomics and metabolomics levels. Metagenome reveals taxonomic composition and functional genes abundance. Metatranscriptome accompany with metaproteome further reflect the temporal fluctuation of gene expression. Metabolome identifies metabolites associated with phenotype and physiology as biomarkers. Previously, biologists focused on one or part of all types of meta-omic information, while the integration of metagenomics, metatranscriptomics, metaproteomics and metabolomics data has begun to gain attention for the purpose of systematically characterizing complex microbial communities [1]. Therefore, related bioinformatics tools for processing all types of meta-omics data is in urgent need.

Though the combination of meta-omics approaches may describe a single microbiota in a systems-level, the functional genomic traits associated to host niches and ecological habitats remains obscure. Therefore, it is necessary to introduce comparative meta-omic methods, which refers to statistically comparing meta-omics data from two or more microbiota samples. During the past decades, comparative meta-omics analysis has been established as a routine procedure applied in human pathology and ecology studies. Researchers have already discovered host-specific genes in human gut microbiotas from comparisons between obese and lean volunteers [2], between long- and short-term dietary volunteers [3, 4] and between patients of nonalcoholic fatty liver disease (NAFLD) [5] or irritable bowel syndrome (IBS) [6] and healthy control volunteers. Meanwhile, by applying these techniques, many studies have reported that the composition of microbial community varies with depth of ocean [7, 8] and oscillates seasonally in Western English Channel [9]. Gene expression pattern of a microbiota fluctuates during different growth stages in Acid Mine Drainage (AMD) [10]. Furthermore, it is notable that an increasing number of studies pay attention on measuring physiological or ecological variables for comprehensively investigating the responds of microbial communities to environmental factor variations $[3,4,7,11,12]$. This trend requires bioinformatics tools not only to distinguish environmental effects on microbiotas through $p$-value from hypothesis testing or correlation analysis but also to unveil intrinsic mechanisms by statistical modeling such as regression analysis.

For comparative metagenomics, the first tool named as XIPE-TOTEC offered two-sample test and utilized metagenomic shotgun sequences as input [13]. Then, MEGAN was designed to perform barplot for comparing multiple samples clustered in taxonomic or functional clustering views and integrate all types of meta-omics data except metabolomics data in the latest version [14]. IMG/M is a web portal supporting a systematical service containing taxonomic classification, sequence assembly, functional annotation and differential abundance analysis for two- and multi-sample comparison of metagenomic reads [15]. Another comparative metagenomics analysis tool, STAMP, mainly exploits Fishers's exact test in two-sample test and $t$-test in two-group samples. [16]. MetaStats, developed for two- and multi-sample comparison was exploited on data normalization for metagenomic data [17]. Later on, FANTOM emphasized its ability in comparison between two groups of metagenomic samples which was implemented with user-friendly graphical interface [18].

Several bioinformatic programs had been developed for comparative metagenomics, however few tools were specialized for metatranscriptomics, metaproteomics and metabolomics data comparison (see Table 1 for details). To compare metatranscriptomics samples, metagenomeSeq were often introduced in $16 \mathrm{~S}$ rRNA, marker-gene expression, RNA-seq data abundance comparison. It was capable for correcting bias caused by variations on sequencing coverage [19]. As for metaproteomics data, MEGAN and STAMP were reported able to process. While only XCMS, an online metabolomic processing platform, performs two-group comparison [20].

Recently, the rapid accumulation of all types of metaomics data brings out three major challenges. Firstly, most comparative analysis tools focused on one type of metaomics data. A universal analysis tool, which is applicable for all types of meta-omics data, will be convenient for researchers characterizing microbiota in multiple metaomics levels. Secondly, all these tools paid no attention to unveil the correlation between microbiota and its living conditions such as temperature, humidity, $\mathrm{pH}$ value and salinity. Lacking of this analysis will definitely hamper biologists from deciphering the microbial adaptive strategy and other interaction between microbes and habitats. Finally, as there are a number of hypothesis testing methods employed in those tools, choosing an optimal one is thus a challenge for users without enough training in statistics. Therefore, an automatical hypothesis testing method selection function based on intrinsic attributes of meta-omics data will greatly improve user experience.

In this study, we present MetaComp, a graphical software incorporates metagenomics, metatranscriptomics, metaproteomics and metabolomics data by accepting abundance profile matrices (APM) saved as txt or BIOM 
Table 1 Input data for available comparative meta-omic tools

\begin{tabular}{|c|c|c|c|c|c|}
\hline Tool & $\begin{array}{l}\text { Meta-omics } \\
\text { Data }^{a}\end{array}$ & Input Format & $\begin{array}{l}\text { Hypothesis } \\
\text { Testing Modes }^{b}\end{array}$ & $\begin{array}{l}\text { Multiple Testing } \\
\text { Correction }^{c}\end{array}$ & Reference \\
\hline XIPE-TOTEC & GS & SEED output, APM format. & TST & Bonferroni and FDR & [13] \\
\hline MEGAN6 & $\begin{array}{l}\text { GM, GR, GS, } \\
\text { TM* }^{*}, \mathrm{TS}^{*} \\
\text { and } P\end{array}$ & $\begin{array}{l}\text { BLAST, RDP classifier, SIN } \\
\text {-A and STAMP outputs; AP } \\
\text {-M in CSV format, BIOM, } \\
\text { DAA and SAM files. }\end{array}$ & NA & NA & [14] \\
\hline IMG/M4 & GR, and GS & $\begin{array}{l}\text { MG-RAST and BLAST out } \\
\text {-puts; APM, BIOM, fasta } \\
\text { and fastq files. }\end{array}$ & MST and TST & NA & [15] \\
\hline STAMP & $\begin{array}{l}\text { GM, GR, GS, } \\
\mathrm{TM}^{*}, \mathrm{TS}^{*} \\
\text { and } \mathrm{P}^{*}\end{array}$ & $\begin{array}{l}\text { MG-RAST, IMG/M, CoMet } \\
\text { and RITA outputs; APM a } \\
\text {-nd BIOM files. }\end{array}$ & $\begin{array}{l}\text { MST, TGST and } \\
\text { TST }\end{array}$ & $\begin{array}{l}\text { Bonferroni, FDR and } \\
\text { Šidák }\end{array}$ & [16] \\
\hline $\begin{array}{l}\text { Metastats and } \\
\text { metagenomeSeq }\end{array}$ & $\begin{array}{l}\text { GM, GR, GS, } \\
\text { TM and TS }\end{array}$ & APM and BIOM files. & MST and TST & Bonferroni and FDR & {$[17,19]$} \\
\hline Fantom & GR, GS & $\begin{array}{l}\text { CAMERA, MG-RAST and } \\
\text { IMG/M outputs. }\end{array}$ & TGST and TST & Bonferroni and FDR & [18] \\
\hline XCMS & B & $\begin{array}{l}\text { cdf, mzData, mzData.XML, } \\
\text { mzXML, netCDF, wiff } \\
\text { and wiff.scan }\end{array}$ & MST and TGST & FDR & {$[20]$} \\
\hline MetaComp & $\begin{array}{l}\text { GM, GR, GS, } \\
\text { TM, TS, P } \\
\text { and B }\end{array}$ & $\begin{array}{l}\text { BLAST, HMMscan, IMG/M, } \\
\text { MG-RAST, MZmine, Kraken } \\
\text { and PhymmBL outputs; APM } \\
\text { and BIOM files. }\end{array}$ & $\begin{array}{l}\text { MST, TGST and } \\
\text { TST }\end{array}$ & Bonferroni and FDR & This work \\
\hline
\end{tabular}

asterisk ${ }^{*}$ ) denotes that the data types are not designed to be processed but compatible with this tool as an input. Abbreviation of meta-omics data types: GM: amplicon sequenced metagenomic marker gene sequeneces; GR: amplicon sequenced 16S rRNA sequences; GS: shotgun sequenced metagenomic sequences; TM: amplicon sequenced metatranscriptomic marker gene sequences; TS: shotgun sequenced metatranscriptomic sequences; P: metaproteomic sequences. B: metabolomic data ${ }^{b}$ Abbreviation of hypothesis testing modes. MST: multi-sample test; TGST: two-group sample test; TST: two-sample test

cFDR denotes for false discovery rate correction

format [21] and the outputs of BLAST [22], HMMER [23], Kraken [24], MG-RAST [25], MZmine [26] and PhymmBL [27] as input. To reveal the interaction between microbial community and its living condition, a novel quantitative characterization of the effect of environmental factors on microbial community through a nonlinear regression is introduced. MetaComp also provides a series of statistical analysis and the visualization for the comparison of functional, physiological and taxonomic signatures in two-, multi- and two-group sample tests. During two-group comparison, MetaComp is able to automatically select the most appropriate hypothesis testing strategy based upon characteristics of the given data set. Moreover, according to our estimation, the selected hypothesis testing method demonstrates the best performance in comparison among mainly used statistical tools. These novel functions agree with the core concerns of comparative meta-omics in this big data era.

\section{Implementation}

MetaComp is implemented in $\mathrm{C \#}$ and $\mathrm{R}$ programming languages. The software installer for Windows system, $\mathrm{R}$ program and databases of $\mathrm{COG}, \mathrm{KO}$ and Pfam categories for Linux system and user guide can be found at the website http://cqb.pku.edu.cn/ZhuLab/ MetaComp/ or at the GitHub site https://github.com/ pzhaipku/MetaComp/. The website of MetaComp provided highlight descriptions, pages about software workflow, convenient download pages, online user guides, detailed demonstration of all application examples with input data and contacts of authors. As illustrated in Fig. 1, MetaComp provides a concise graphical user interface that two drop-down menus are presented: File (for data input) and Analysis (for analysis method selection). In the following subsections, we first review the preparation of abundance profiles for four types of meta-omics data. Then, based on outputs of these pipelines, we further introduce the various standard input formats for 


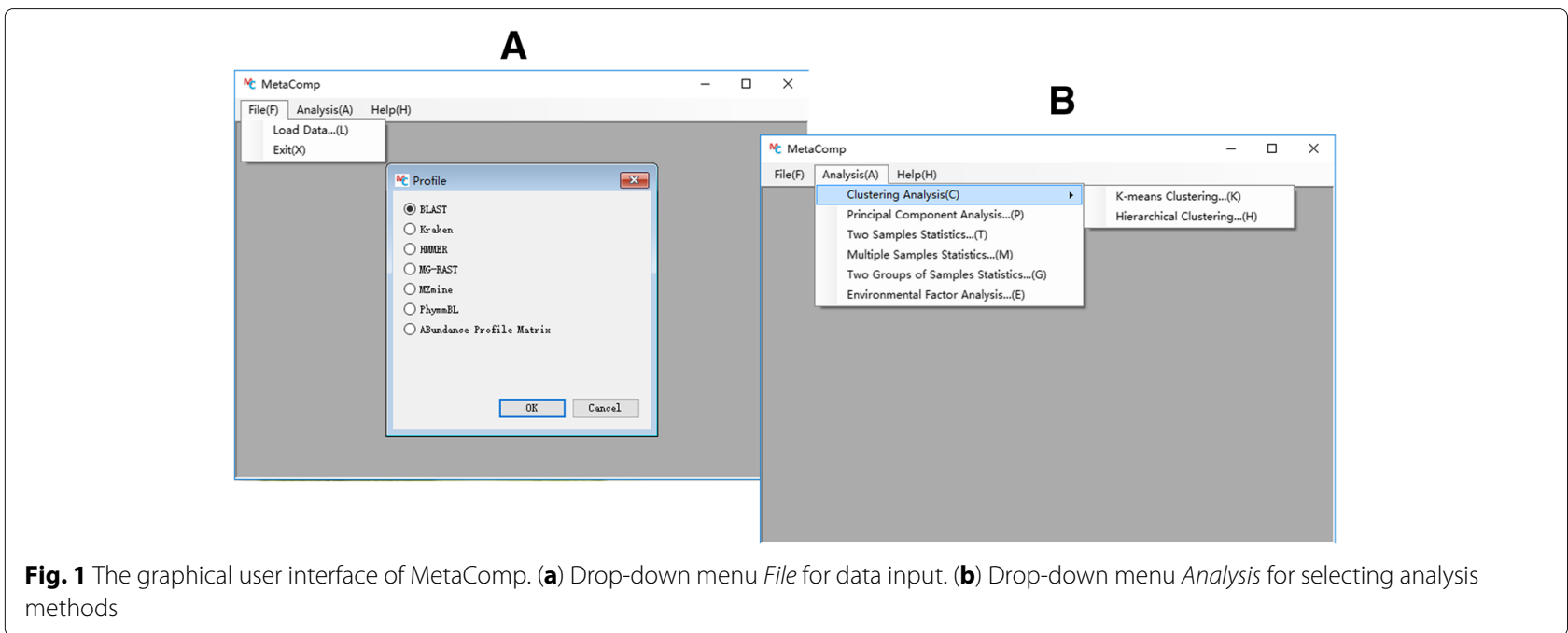

MetaComp. Finally, integrated statistical analysis options and visualization for these analysis are demonstrated. The structure as well as work flow of MetaComp is displayed in Fig. 2.

\section{Preparation of abundance profiles of meta-omics data}

According to Fig. 3, three types of macromolecules and other metabolites are first extracted from environmental samples separately then sequenced or measured by different techniques. Two major sequencing strategies for DNA and RNA chains are designed in different purposes. Shotgun sequencing is aiming to reflect the global content of metagenome or metatranscriptome by randomly amplifying and sequencing all DNA or RNA sequences, while amplicon sequencing is focused on selected marker genes or 16S rRNA by specifically amplifying primer induced sequences $[25,28]$. The metaproteome and metabolome are measured in another routine. Proteins and metabolites are first separated and fractionized by multidimensional liquid chromatography (LC) then measured by tandem

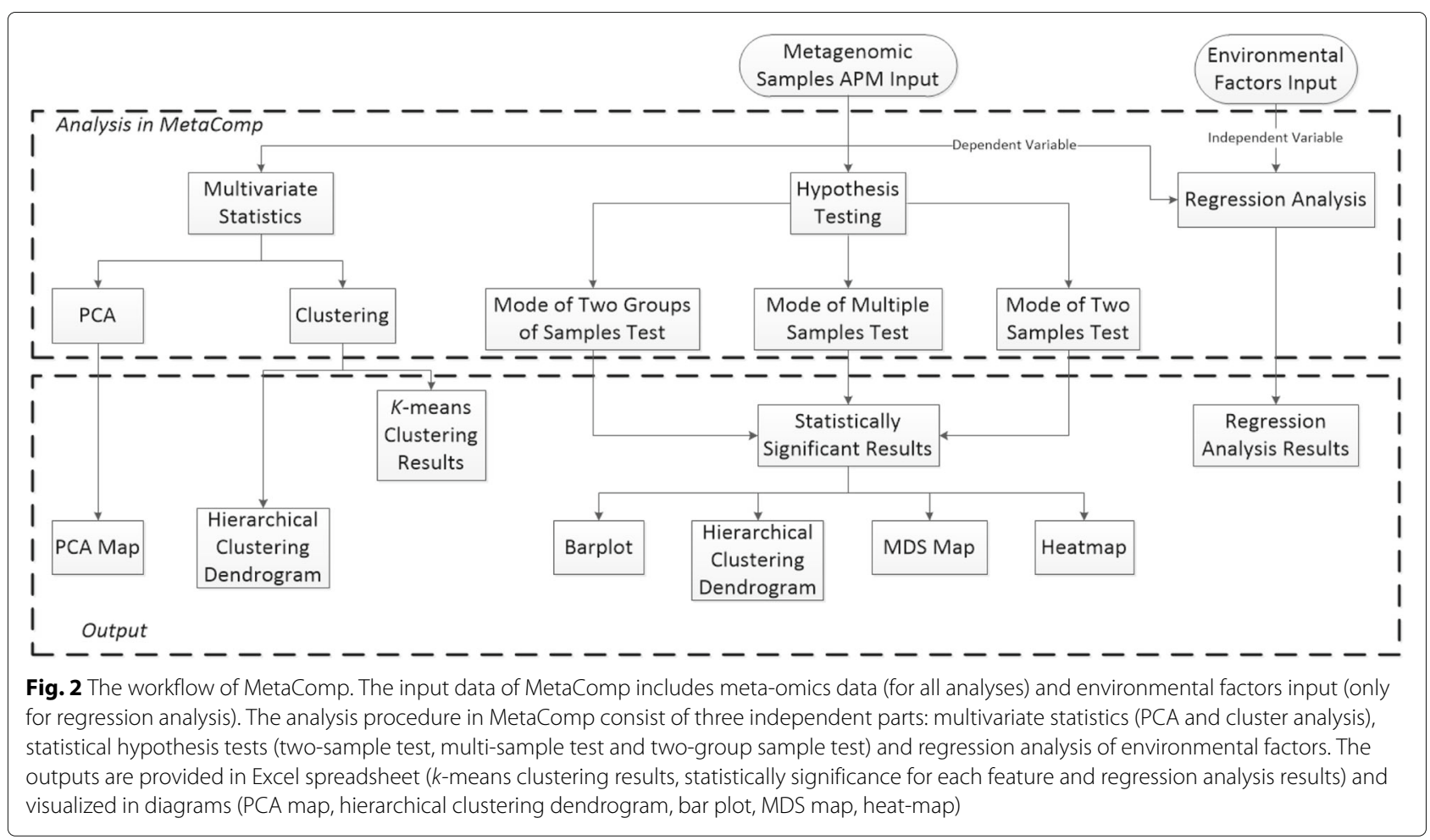




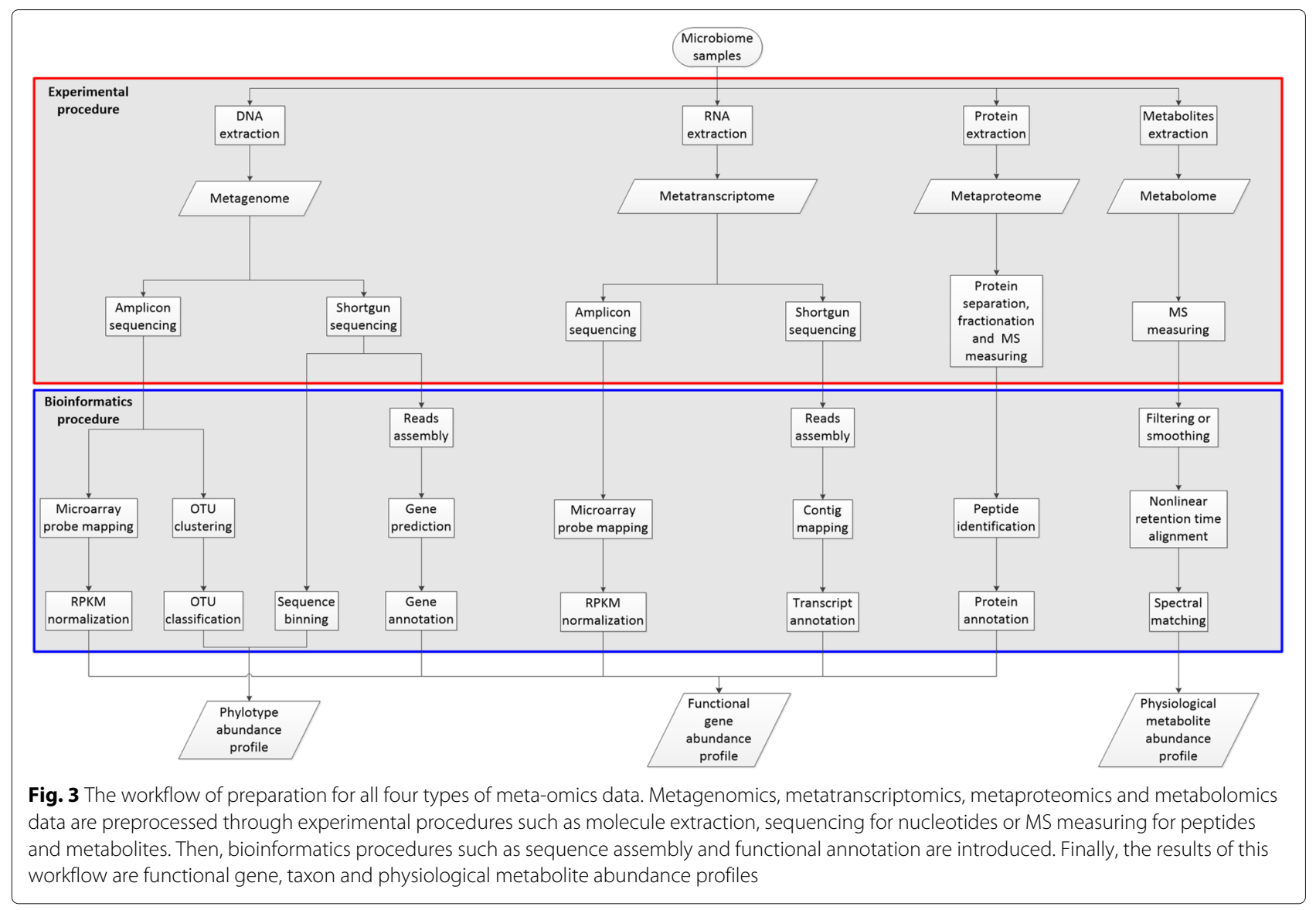

mass spectrometer and the final result is mass spectrometry (MS) spectra data $[20,29,30]$.

After these experimental processing, the rest procedures for functional gene, taxon and physiological metabolite abundance profiling within a sample are conducted mainly by bioinformatics approaches. There are three major workflows for generalizing functional gene abundance profiles from meta-omics data. The workflow for metagenomics and metatranscriptomics amplicon sequencing data are directly mapped to marker genes through microarray techniques, and after reads per kilobases million (RPKM) normalization or other complicated normalization the gene abundance profiles are obtained.

To extract taxon profile of a metagenomic sample, both $16 \mathrm{~S}$ rRNA reads and binning results are utilized. The reads of amplicon sequenced $16 \mathrm{~S}$ rRNA are primarily clustered into operational taxonomic units (OTUs), then each OTU is classified using RDP classifier [31], QIIME [32], Mothur [33] or just BLAST against taxonomic 16S rRNA databases (RDP [34], Greengenes [35], SILVA [36] and NCBI 16S rRNA). Except this procedure, shotgun sequenced genomic reads carry phylogenetic features as well. Based on characterizing nucleotide composition of a read or aligning to reference genomes, a series of approaches denoted as binning are developed. Among these approaches, PhymmBL is the most accurate method, and recently software Kraken achieves a comparable accuracy but consumes less time.

The profiling of shotgun sequencing metageomics data is consist of three steps: reads assembly, gene prediction and gene annotation. DNA reads are first assembled into contigs or scaffolds through IDBA-UD [37], CABOG [38], MAP [39] or InteMAP [40]. After that, MetaGeneMark [41], Glimmer-MG [42] or MetaGUN integrated with MetaTISA [43, 44] are adopted for gene prediction. MetaGeneMark [41] and Glimmer-MG [42] are able to perform a solid detection for known coding genes within metagenomic contigs, while MetaGUN further enables to discover novel genes through domain based searching strategy [43]. At last, by utilizing BLAST, HMMER or MG-RAST to search in ontology databases including COG [45], KO [46], Pfam [47] and SEED [48], the functional profile for metagenomics data is obtained.

Though the processing of shotgun sequenced metatranscriptomics data is consist of three steps as well, the second step of transcriptomic analysis is contig mapping other than gene prediction. After assembled by trinity 
[49], RNA contigs and scaffolds are simply mapped to reference genomes or Uniprot database [50] utilizing BWA [51] or Bowtie [52] program. The functional profile is obtained in the same way as that for metageomics data.

The LC-MS measured metaproteomics data are profiled in just two steps: peptide identification and protein annotation. As for peptide identification step, MS data are matched with amino acid or nucleotide sequences via search engines such as SEQUEST [53] and Mascot [54]. Then, it shares the same functional annotation step with metagenomic and metatranscriptomic analysis.

The physiological biomarker reflected by metabolomics data are detected in a unique procedure and consist of tandem MS data filtering or smoothing, nonlinear retention time alignment of peaks and spectral matching of the tandem MS data to METLIN [55] and MassBank [56] databases. This pipeline can be realized by MZmine [26] and XCMS [20] tools, resulting in fully annotated MS profiles of metabolites.

\section{Standard input formats}

Though the output file formats of all these mentioned softwares are largely different, they are regarded as standard inputs of MetaComp. The functional abundance profiling are mainly conducted by BLAST and HMMER at the last annotation step, and only a few meta-omics data are offered in tab separated variables form as MG-RAST. For taxon abundance profiling, many OTU clustering programs (e.g. QIIME, Mothur and RDP classifier) employ BIOM format files as output, meanwhile binning programs always offer simply two column hit results. Besides, the output of physiological biomarker detection is always arranged in a tabular format such as MZmine. After loaded, input files are automatically transferred into APM whose rows correspond to features and columns correspond to individual meta-omic samples. Moreover multiple file selection is supported. Here, the features refer to functional gene categories or phylotype categories. The total number of features $i\left(F_{i}\right)$ observed in metagenomic sample $j\left(S_{j}\right)$ is represented by $c_{i j}$ (see Table 2 ).

\section{Statistical analysis options and visualization}

We integrated a series of statistical analysis options in MetaComp (see Fig. 2), ranging from descriptive multivariate statistical analyses, hypothesis testing analyses,

Table 2 Input data of MetaComp

\begin{tabular}{lllll}
\hline & $S_{1}$ & $S_{2}$ & $\ldots$ & $S_{n}$ \\
\hline$F_{1}$ & $c_{11}$ & $c_{21}$ & $\ldots$ & $c_{n 1}$ \\
$F_{2}$ & $c_{12}$ & $c_{22}$ & $\ldots$ & $c_{n 2}$ \\
$F_{3}$ & $c_{13}$ & $c_{23}$ & $\ldots$ & $c_{n 3}$ \\
$\ldots$ & $\ldots$ & $\ldots$ & $\ldots$ & $\ldots$ \\
$F_{m}$ & $c_{1 m}$ & $c_{2 m}$ & $\ldots$ & $c_{n m}$ \\
\hline
\end{tabular}

nonlinear regression analysis of environmental factors and corresponded visualization. Herein, we introduce each statistical analysis option in the following paragraphs.

\section{Multivariate statistics}

MetaComp employs principal component analysis (PCA) and clustering approaches (e.g. $k$-means clustering and hierarchical clustering) to present an overview of the differences among the given sets of meta-omics samples and highlight main features for each sample. Though it is a descriptive statistical function, these results are indispensable visualizations of meta-omics features. For example, enterotypes is illustrated by PCA figure.

\section{Statistical hypothesis tests}

Statistical hypothesis tests for comparative meta-omics are provided in MetaComp through three test modes:

- Mode of two-sample test: As the amount of metaomic features is usually huge, we choose $z$-test instead of $t$-test as our default method to assess statistical significant differences between two individual samples. Thus $z$-score for the feature $F_{i}$ is read as

$$
z_{i}=\left(\frac{c_{i 1}}{N_{i 1}}+\frac{c_{i 2}}{N_{i 2}}\right) / \sqrt{P(1-P)\left(\frac{1}{N_{i 1}}+\frac{1}{N_{i 2}}\right)},
$$

where $N_{i 1}=\sum_{i=1}^{m} c_{i 1}, N_{i 2}=\sum_{i=1}^{m} c_{i 2}$ and $P=\left(c_{i 1}+c_{i 2}\right) /\left(N_{i 1}+N_{i 2}\right)$. Since $z$-test is not valid if the feature size is insufficient, the prerequisite of $z$-test is $\min \left(c_{i 1}, c_{i 2}\right) \leqslant z_{i}^{2}$. When the sample size is small or user demands a more strict hypothesis testing method, MetaComp also offers Fisher's exact test as an alteration (see the user guide of MetaComp for detailed recommendation).

- Mode of multi-sample test: In this mode, pairwise tests between all conceivable pairs of samples are executed by $z$-test. The $p$-value of a specific feature $i$ is the minimum of all conceivable $p$-values. Thus we can identify that the selected feature is significantly different in at least one pair of samples.

- Mode of two-group sample test: During this test, all samples are classified into two groups. In MetaComp, we provide four statistical hypothesis test methods ( $t$-test, paired $t$-test, Mann-Whitney $U$ test and Wilcoxon signed-rank test) to assess whether a specific feature is significantly different between two groups of samples. Users can choose a proper method themselves or let MetaComp determine the most suitable test method according to the criterion shown in Table 3.

If MetaComp judges that input data follow a Gaussian distribution, parametric hypothesis testing should be introduced. Otherwise when sample size is small or 
Table 3 Criterion for selecting appropriate test

\begin{tabular}{lll}
\hline & Parametric & Non-parametric \\
\hline Independent & t-test & Mann-Whitney $U$ test \\
Correlated & Paired $t$-test & Wilcoxon signed-rank test \\
\hline
\end{tabular}

normality assumption is violated, nonparametric hypothesis testing should be conducted. If two groups of samples are consist of matched pairs for resemble units, or one group of units that has been tested twice, it indicates that two groups of samples are correlated. This automatical selection will be helpful for users lacking of adequate statistical training. Moreover, odds ratio (OR) test was also implemented to evaluate the relative abundance for each feature as Table 4 demonstrated.

Here, $G_{1}$ and $G_{2}$ is in short for Group 1 and Group 2. $c_{j k}$ denotes as counts for the $j$-th feature from the $k$-th group samples.

Considering the possibility of unevenness between two groups, an empirical continuity correction has been introduced to improve the accuracy of the test. Consequently, OR statistic for feature $i$ is

$$
\log _{2} \text { OR }(i)=\log _{2} \frac{\left(M_{11}+\frac{R}{R+1}\right)\left(M_{22}+\frac{1}{R+1}\right)}{\left(M_{12}+\frac{1}{R+1}\right)\left(M_{21}+\frac{R}{R+1}\right)} .
$$

Where $R=M_{1} / M_{2}$. According to the formula above, features are categorized as group 1 enrichment (when $\log _{2} O R(i)>1$ ) or group 1 scarcity (when $\log _{2}$ OR $\left.(i)<1\right)$.

\section{Multiple test correction}

As the typical meta-omics profile consists of hundreds to thousands of features (e.g. Pfam/COG functional profiles), direct application of statistical method described above may probably lead to large numbers of false positives. For example, choosing a threshold of 0.05 will introduce 500 false positives in a profile contains 10000 features. Therefore, two correction methods are implemented in the MetaComp software to solve this problem, including false discovery rate (FDR) as the default option and a stricter option Bonferroni correction.

Table 4 Contingency table for odd ratio test

\begin{tabular}{llll}
\hline$G_{1}$ & $G_{2}$ & Sum \\
\hline$F_{j j=i}$ & $M_{11}=\sum_{j \in G_{1}} c_{j 1}$ & $M_{12}=\sum_{j \in G_{2}} c_{j 2}$ & $n_{1}=\sum_{l=1}^{2} M_{1 k}$ \\
$F_{j j \neq i}$ & $M_{21}=\sum_{j \notin G_{1}} c_{j 1}$ & $M_{22}=\sum_{j \notin G_{2}} c_{j 2}$ & $n_{2}=\sum_{l=1}^{2} M_{2 k}$ \\
Sum & $M_{1}=\sum_{j=1}^{2} M_{j 1}$ & $M_{2}=\sum_{j=1}^{2} M_{j 2}$ &
\end{tabular}

\section{Regression analysis of environmental factors}

MetaComp provides a novel function, regression analysis of environmental factors, which means regression analysis of the influence exerted by environmental factors on microbial communities. This original function is implemented by nonlinear regression analysis via the lasso algorithm. MetaComp first normalizes the data of both meta-omics samples and environmental factors. After that, the $i$ th environmental factor in $j$ th sample (which we shall denote by $x_{i j}$ ) is considered as independent variable, and the $j$ th frequency of $k$ th feature (which we shall denote by $y_{k j}$ ) is considered as dependent variable. Therefore, the regression function is:

$$
y_{k j}=\sum_{i} \alpha_{k i} x_{i j}+\sum_{m, n}^{m \neq n} \beta_{k m n} x_{m j} \cdot x_{n j}
$$

where $x_{m j} \cdot x_{n j}$ means the co-effect of environmental factor $x_{m j}$ and $x_{n j}$ to feature $y_{k j}$. Then, $\alpha_{k i}$ and $\beta_{k m n}$ represent the regression coefficient of the function. For any specific feature, the influence of environmental factors on samples is appraised by coefficient value and correlation value. Moreover, the reliability of the regression coefficient is estimated by $p$-value. Only when all $p$-values meet the prescribed standard, the result of regression would be accepted by MetaComp.

\section{Visualization of statistical significance analysis}

For the MetaComp software, the visualizations of the hypothesis testing results are displayed in Fig. 4, including:

- Bar plot: Bar plot is exhibited for the top 10 significantly different features with their frequencies in each sample.

- Hierarchical clustering dendrogram and multi-dimensional scaling map: Hierarchical clustering dendrogram and multi-dimensional scaling map are presented to illustrate the clustering and distance information of meta-omics samples respectively. Features with significant differences $(p<0.05)$ are involved in this clustering.

- Two-dimensional heat-map: Two-dimensional heat-map is performed to investigate the relative abundance of each feature and the similarity among independent samples.

Moreover, our software enables to save the figures in many formats (e.g. .eps, .pdf, .png and .jpeg etc.) that can be used directly for publication.

\section{Results and discussion Analysis process}

The analysis workflow of MetaComp can be described as follows (see Fig. 2 for a graphical overview): 

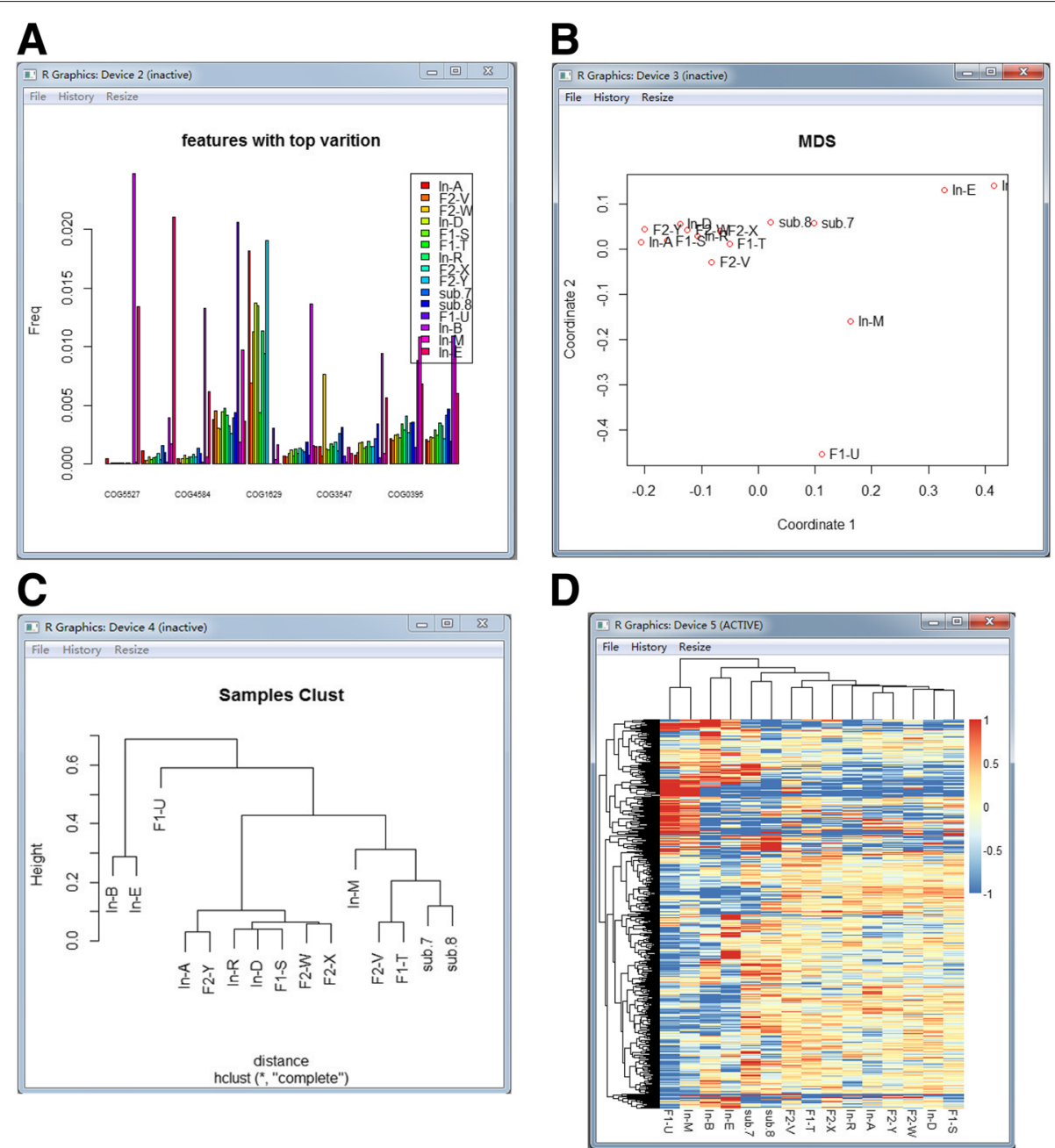

Fig. 4 The visualization examples of MetaComp. a The bar plot of the top ten significantly different features. $\mathbf{b}$ The multi-dimensional scaling map of samples. Each point represents an individual sample. c The hierarchical clustering dendrogram of given samples. $\mathbf{d}$ The heat-map of given samples

- Meta-omics input data are loaded for the further statistical processing through File menu. Outputs of BLAST, HMMER, Kraken, MG-RAST, MZmine and PhymmBL, BIOM format and APM saved as txt files are able to load by MetaComp. Additional environmental factors input data are required if users intend to conduct environmental factors analysis on APMs of samples. These environmental factors are also arranged as APMs.

- After loading input data, users should choose an analysis from multivariate statistics, statistical hypothesis tests and environmental factor analysis. The option is made through Analysis menu and parameters is set in pop-up dialog boxes.

- The result of analysis is displayed as Excel spreadsheet with corresponding visualization.

\section{Application in comparison of meta-omic samples}

There are four types of meta-omics data characterizing microbiota in different levels but revealing two types of information-static composition of taxon as well as functional gene and dynamic gene expression condition of a microbial community. Metagenomics data including $16 \mathrm{~S}$ rRNA sequences provide an overview of both phylogenetic and functional gene composition, however metatranscriptomics, metaproteomics and metabolomics data decipher the functional response of a microbiota to various environmental perturbations over spatial and temporal scales. Particularly, metatranscriptome and metaproteome are quite similar and aiming to reflect the fluctuation of functional gene expression, meanwhile metabolome complement with metabolic flux variations of biological pathways via specific physiological 
biomarkers to unveil the functional gene regulation indirectly. Metagenomics data provide the universe of all possible protein coding genes and metabolic pathways, meanwhile metatranscriptomics, metaproteomics and metabolomics data identify a subsets of active genes and pathways under specific environment. Besides, according to our application of MetaComp on various types of meta-omics data, though these techniques characterize microbiome in different levels and may introduce concentration instead of abundance or frequency, it seems not result in differences on the features of data itself. Hereafter, we demonstrate that the application of MetaComp in meta-omics data presenting in both compositional and dynamical characterizations.

\section{Example 1. eight typical environmental metagenomic samples}

Herein we analysed eight typical environmental metagenomic samples, including whale fall, Sargasso Sea, Minnesota farm soil and AMD, which were originally compared by Tringe et al. [57] (input data are listed in Additional file 1: Table S1). The input shotgun sequenced data was annotated by Pfam database. Though amplicon sequenced $16 \mathrm{~S}$ rRNA data was not included in this example, the processing was all the same as for shotgun sequenced metagenomic data. So that we only focused on comparing shotgun sequenced data in this case. During this analysis, we chose multi-sample test and the results clearly illustrate that the protein family profile of a microbial community is similar to that of other communities when their living environments are highly analogous (illustrated in Fig. 5). According to the detailed analysis results demonstrated in Additional file 2: Table S2, 3456 protein families are significantly different (FDR $<0.01$ ) among all given 11,110 compared protein families. These different features are closely related to the living conditions of metagenomic samples. For example, a large amount of bacteriorhodopsin-like proteins (e.g. PF01036) are found in all three Sargasso Sea samples, while these proteins are hardly detected in other samples. This protein is involved in obtaining light energy. In addition, since the content of potassium is apparently higher in AMD and soil, the quantity of potassium ion channel protein (e.g. PF03814, PF02705) in AMD and Minnesota farm soil greatly surpasses that in other samples (shown in Table 5).

\section{Example 2. Acid Mine Drainage metaproteomic samples}

Due to similarity on characterizing dynamics of functional gene expression in a microbiota, it is enough to choose either metatranscriptomic samples or metaproteomic samples to test MetaComp performance. We then take metaproteomic samples of membrane and cytoplas-

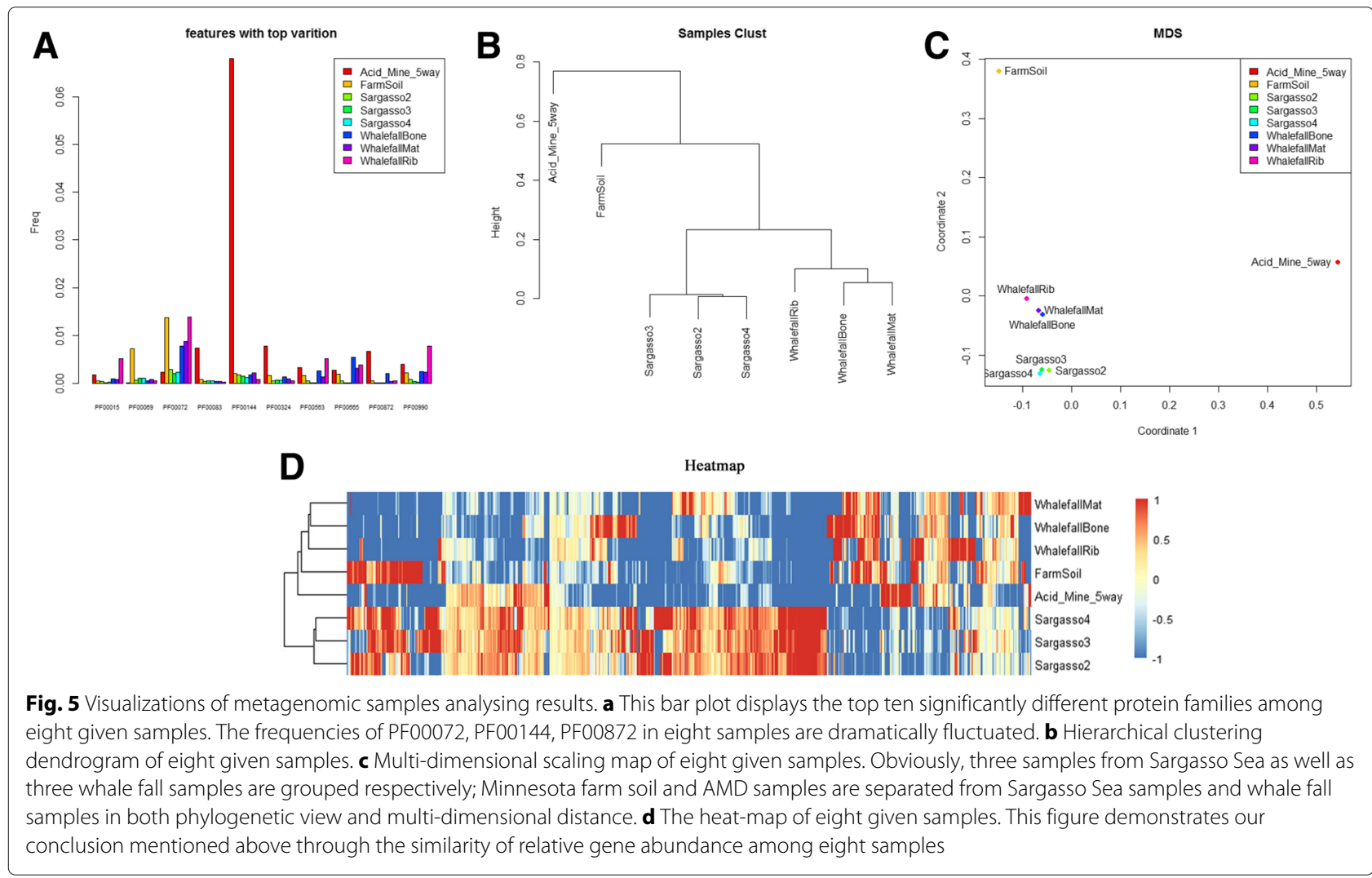


Table 5 Part of whale fall, Acid Mine Drainage, Sargasso Sea, and Minnesota soil metagenomic samples analysis result

\begin{tabular}{|c|c|c|c|c|c|c|c|c|c|c|c|}
\hline & AMD & Soil & $\mathrm{S} .2^{\mathrm{a}}$ & $\mathrm{S} .3$ & S.4 & W.Bone ${ }^{b}$ & W.Mat & W.Rib & $p$-value & $q$-value & Function \\
\hline PF01036 & 0 & 1 & 344 & 354 & 332 & 0 & 0 & 0 & $1.60 \mathrm{e}-35$ & $4.67 e-34$ & Bacteriorhodopsin-like protein \\
\hline PF03814 & 99 & 17 & 3 & 4 & 7 & 0 & 0 & 1 & $5.22 \mathrm{e}-48$ & $2.92 \mathrm{e}-46$ & $\begin{array}{l}\text { Ion channel KdpA Potassium-transporting } \\
\text { ATPase A subunit }\end{array}$ \\
\hline PF02705 & 0 & 87 & 15 & 30 & 30 & 10 & 0 & 1 & $2.27 e-56$ & $3.59 \mathrm{e}-54$ & APC K trans $\mathrm{K}^{+}$potassium transporter \\
\hline PF01077 & 42 & 4870 & 62 & 51 & 71 & 57 & 45 & 37 & 0 & 0 & $\begin{array}{l}\text { NIR_SIR Nitrite and sulphite reductase } 4 \mathrm{Fe}-4 \mathrm{~S} \\
\text { domain }\end{array}$ \\
\hline
\end{tabular}

mic proteins from biofilms at B-drift site of Richmond mine as input data for MetaComp. The biofilms were classified into early (labeled as GS0), intermediate (labeled as GS1) and late (labeled as GS2) growth stages. Significantly correlated proteins were identified by significance analysis of microarrays (SAM) or clustered by self-organizing tree algorithm (SOTA) in previous study (see Additional file 3: Table S3 for more details) [10]. Since MetaComp is designed for count data which means no negative variables is allowed as input, we transformed the original relative abundance data exponentially, with the base as 10 .

Herein, we conducted two-sample $z$-test for these three samples. The results agree with the previous classification in most cases. For instance, $91.9 \%$ of early growth stage, $93.2 \%$ of late growth stage and $83.3 \%$ of intermediate growth stage expressed genes identified either by SAM or SOTA are also recognized by MetaComp. In addition, the rest proteins cannot provide comparing result due to too low abundance among compared samples.

We further observed that abundance of 65 out of 144 proteins identified previously as early stage expressed demonstrate significantly lower $(p<0.05)$ in early growth stage than intermediate stage. Meanwhile, previously identified intermediate stage expressed proteins indicate a $p$-value less than or equal to $4.18 \times 10^{-30}$. With this $p$-value as threshold, 19 proteins still express significantly larger in intermediate stage than early stage, within which 10 proteins are engaged in environmental sensing procedure, others also correspond with specific cell processing and metabolic processing (see Additional file 4: Table S4 and Additional file 5: Figure S1 to S3 for more details). For example, LeptoII_Cont_10776_GENE_10 annotated as an important heat shock protein-GroEL, is regulated by RNA polymerase subunit $\sigma^{32}$ during heat stress [58]. LeptoII_Scaffold_8241_GENE_340 annotated as Acetyl-CoA synthetase is also demanded in stationary phase rather than exponential phase to reduce fatty acids generated from membrane lipids [59]. Moreover, flagella synthesis related proteins (LeptoII_Scaffold_8241_GENE_209 annotated as FlgD, LeptoII_Scaffold_8241_GENE_653 annotated as FliD and LeptoII_Scaffold_7904_GENE_5 annotated as FlhA) are classified as intermediate expressed protein by MetaComp. According to the previous results [10], other flagellar proteins are expressed during intermediate and late stages of growth. We further noticed that LeptoII_Scaffold_8241_GENE_209, LeptoII_Scaffold_82 -41_GENE_653 and LeptoII_Scaffold_7904_GENE_5 only take parts in middle procedures of flagella biosynthesis other than from the beginning procedures [60]. Therefore, these genes identified as mainly expressed in intermediate stage by MetaComp is reasonable (these genes are listed in Table 6).

Table 6 Part of early and intermediate stage gene analysis result

\begin{tabular}{|c|c|c|c|c|c|c|}
\hline Protein ID & $\mathrm{KO}$ & $\begin{array}{l}\text { Early } \\
\text { stage }\end{array}$ & $\begin{array}{l}\text { Intermediate } \\
\text { stage }\end{array}$ & $p$-value & Function & Annotation \\
\hline Leptoll_Cont_10776_GENE_10 & K04077 & 2.38 & 6.94 & $4.88 \mathrm{e}-32$ & $\begin{array}{l}\text { Cellular } \\
\text { Processing }\end{array}$ & Chaperonin GroEL \\
\hline Leptoll_Scaffold_8241_GENE_340 & K01895 & 1.96 & 6.35 & $3.07 e-30$ & $\begin{array}{l}\text { Environmental } \\
\text { sensing }\end{array}$ & Acetyl-CoA synthetase \\
\hline Leptoll_Scaffold_8241_GENE_209 & K02389 & 2.57 & 6.78 & $1.72 \mathrm{e}-30$ & $\begin{array}{l}\text { Environmental } \\
\text { sensing }\end{array}$ & $\begin{array}{l}\text { Probable flagellar hook capping protein } \\
\text { (FlgD) }\end{array}$ \\
\hline Leptoll_Scaffold_8241_GENE_653 & K02407 & 1.32 & 7.84 & $1.36 \mathrm{e}-41$ & $\begin{array}{l}\text { Environmental } \\
\text { sensing }\end{array}$ & $\begin{array}{l}\text { Putative flagellar hook-associated protein } \\
\text { (FliD) }\end{array}$ \\
\hline Leptoll_Scaffold_7904_GENE_5 & K02400 & 0.77 & 7.63 & $4.51 \mathrm{e}-43$ & $\begin{array}{l}\text { Environmental } \\
\text { sensing }\end{array}$ & Probable flagellar biosynthesis protein FlhA \\
\hline
\end{tabular}




\section{Example 3. human fecal metabolomic samples}

Since metabolome data indirectly reflect the conditional response of a microbial community, which is distinct with metatranscriptome and metaproteome, it is necessary to examine the performance of MetaComp on this data. We applied MetaComp on metabolomics data of fecal microbiota detected by Raman et al. [5]. The original data includes two groups of samples: 30 NAFLD patients for one group and another group with an equal number of healthy volunteers (see Additional file 6: Table S5 for more details). In Raman's study, researchers focused on detecting volatile organic compounds (VOC) which may exert toxic effect to human liver and secreted by human gut microbiota [5]. VOCs were not quantitatively measured but counted by detected or not per individual. Therefore, by gathering this binary counts for both prevalence group and control group, the maximum value for each type of VOC per group is 30 .

MetaComp conducted a two-sample $z$-test on NAFLD and control group, and results indicate that 15 out of 220 VOCs are significantly different between two groups (see Additional file 7: Table S6 and Additional file 5: Figure S4 for more details). Furthermore, most VOCs identified as significantly different are included in previous study expect indolizine and acetic acid butyl ester and it may because of lacking of hits (only 5 hits in appeared in healthy control samples) that makes it difficult to be detected in previous study. It is notable that 6 out of 8 VOCs enriched in NAFLD fecal samples are short fatty acid esters. These derivatives of short fatty acids reflect that a relatively high concentration of hexose dietary such as frequently drinking soft drinks with fructose, which is a cause of NAFLD (shown in Table 7) [61].

\section{Application in regression analysis of environmental factors Example 1. Hawaii Ocean metagenomic samples}

We applied the novel function of MetaComp, regression analysis of environmental factors, on metagenomic samples from Hawaii Ocean [7] (see Additional file 8: Table S7 for more details). The input reads were aligned against COG database [45]. The selected environmental factors are dissolved inorganic phosphate (DIP) and

Table 7 Part of nonalcoholic fatty liver disease samples analysis result

\begin{tabular}{llll}
\hline Compound & Control & NAFLD $^{\text {a }}$ & p-value \\
\hline Butanoic acid, propyl ester & 1 & 14 & 0.0016 \\
Ethyl acetate & 0 & 9 & 0.0045 \\
Acetic acid, pentyl ester & 1 & 10 & 0.0112 \\
Propanoic acid, propyl ester & 5 & 18 & 0.0142 \\
Butanoic acid, 3-methyl-, butyl ester & 1 & 9 & 0.0183 \\
\hline aNAFLD=nonalcoholic fatty liver disease & & &
\end{tabular}

oxygen content (OC) (see Additional file 9: Table S8 for more details). Concluded from the detailed analysis results (see Additional file 10: Table S9 for more details), we discover 102 out of 4,873 COGs which are probably corresponding to the living environment of Hawaii Ocean $(p<0.1)$. Moreover, some of the selected COG features are related to the generation and consumption of Adenosine Triphosphate (ATP), which is evidently related to phosphate and oxygen. For instance, COG0378, as a $\mathrm{Ni}^{2+}$-binding GTPase involved in regulation of expression and maturation of urease and hydrogenase, will generate organic phosphorus as well as dehydrogenase. Moreover, this reaction may consume oxygen. It is obvious that this COG is linked to both DIP and OC. Details of these protein families are shown in Table 8 . Meanwhile, COGs relevant to the content of DIP (e.g. COG0379, COG0458, COG0486, COG0849, COG1190 and COG1921) are selected by MetaComp (illustrated in Fig. 6).

\section{Example 2. Acid Mine Drainage metagenomic samples}

A total of 40 AMD samples distinct in environmental characteristics were previously collected across Southeast China. Sampling procedure and data processing were described previously [62] (see Additional file 11: Table S10 for more details). The measured environmental factors were dissolved oxygen (DO), total organic carbon (TOC) and $\mathrm{SO}_{4}^{2-}$ (see Additional file 12: Table S11 for more details). According to the detailed analysis results (see Additional file 13: Table S12 for more details), we discover 69 out of 142 genes which are presumably related to the living environment $(p<0.1)$. Among the selected genes, fumarate and nitrate reductase (fnr) gene is related to the respiratory chain of bacteria and the reaction of it requires a mass of sulfur. Therefore the abundance of fnr is apparently linked to $\mathrm{DO}$ and $\mathrm{SO}_{4}^{2-}$. Furthermore, ammonia monooxygenase $\mathrm{A}(\mathrm{amoA})$ is an enzyme, which catalyses nitration reaction. This reaction may consume organic carbon and oxygen. It is manifestly related to the content of TOC and DO.

\section{Compared with other tools in differentially abundant features detection}

Variations embedded in meta-omics are always difficult to recognize when the hit number of a feature is slightly different from one group of samples to another but evidently fluctuated among samples of the same group. Since that, to evaluate the differentially abundant features detection ability of current comparative meta-omics methods, we simulated two groups of count data from twin gut samples [2], of which 1,649,149 hits for 1000 COG features was annotated through BLAST against COG database, to reserve the complexity of true data. Then we take a previous evaluation study for reference [63], samples are 
Table 8 Part of relationship between metagenomic samples and environmental factors analysis result

\begin{tabular}{lllllll}
\hline & \multicolumn{3}{c}{ Coefficient } & p-value & Correlation & Annotation \\
\cline { 2 - 4 } & DIP & OC $^{\mathrm{b}}$ & DIP\&OC & & & \\
\hline COG0378 & 0 & 0 & $3.303 \mathrm{e}-06$ & $2.97 \mathrm{e}-02$ & $9.01 \mathrm{e}-01$ & $\mathrm{Ni}^{2+}$-binding GTPase involved in regulation of expression and mature \\
COG1921 & $1.157 \mathrm{e}-03$ & 0 & 0 & $8.41 \mathrm{e}-02$ & $8.22 \mathrm{e}-01$ & Selenocysteine synthase [seryl-tRNASer selenium transferase] \\
COG0318 & $4.841 \mathrm{e}-03$ & 0 & 0 & $8.97 \mathrm{e}-02$ & $8.15 \mathrm{e}-01$ & Acyl-CoA synthetases (AMP-forming)/AMP-acid ligases II \\
\hline
\end{tabular}

${ }^{\mathrm{a} D I P}=$ dissolved inorganic phosphate

${ }^{b}$ Oxygen=oxygen content

first amplified with fold change $q=1.25$ and resampled into two equal sized groups of samples through randomly sampling without replacement. After that, 10\% of COGs from the second group were chosen by chance and downsampled according to binomial distribution. Herein, we denoted $x_{i j}^{\prime}$ as hits of the $j$-th feature from the $i$-th sample and it followed binomial distribution $B\left(x_{i j}, p\right)$, where $x_{i j}$ was hit number after resampling and $p=1 / q$ to control the alteration between two groups. Therefore, we obtained two groups of samples with hits of $1,800,000$ and 1,744,981 in total, respectively. The dataset generated from resampling is demonstrated in Additional file 14: Table S13.

Since $t$-test (employed by Fantom, STAMP, Metastats, XCMS and MetaComp in two-group sample test), paired $t$-test (employed by XCMS and MetaComp in two-group
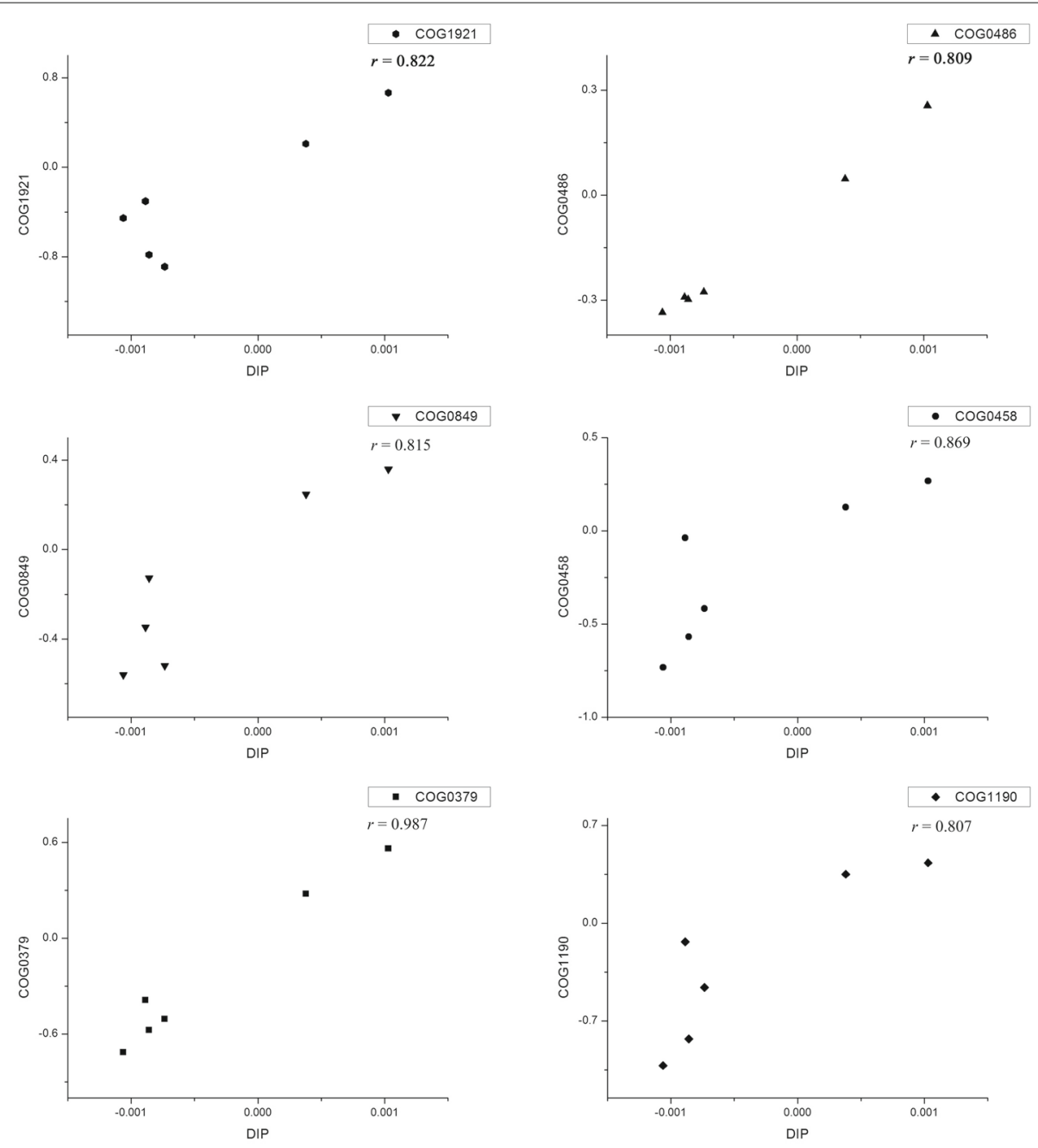

Fig. 6 Diagrams of regression. These diagrams exhibit the relationship between DIP and selected functional genes categorized by COG (COG0379, COG0458, COG0486, COG0849, COG1 190 and COG1921). It is obviously that the abundance of these genes is linear with the content of DIP 
sample test for correlated samples), non-parametric $t$ test (employed by STAMP in two-group sample test), Wilcoxon Mann-Whitney $U$ test (employed by XCMS and MetaComp in two-group sample test for independent samples) and Wilcoxon signed-rank test (employed by XCMS and MetaComp in two-group sample test for correlated samples) were mainly employed hypothesis testing methods, we took these methods for camparison. The result is listed in Table 9, and the detailed significance detection are presented in Additional file 15: Table S14. Under the threshold of FDR $<0.05$, it is obvious that MetaComp automatically adopted Wilcoxon signed-rank test performed the best over other methods with the highest sensitivity (100.0\%) and a decent specificity (99.9\%). Here, sensitivity (SN) and specificity (SP) were calculated with true positive (TP), true negative (TN), false positive $(\mathrm{FP})$ and false negative (FN) as:

$$
S N=\frac{T P}{T P+F N}, \quad S P=\frac{T N}{T N+F P} .
$$

We further plotted the Receiver operating characteristic (ROC) curve (shown in Fig. 7) to demonstrate the performance of all five hypothesis testing methods as well. This analysis indicated that area under ROC curve (AUC) was almost 1.0 and confirmed automatical selection (other than recommended by XCMS) of MetaComp was the most appropriate one.

\section{Conclusion}

Compared with the previously developed tools, MetaComp takes advantages in three fields.

- Our software is universally applied in all types of meta-omics data including metagenomics, metatranscriptomics, metaproteomics and meta-bolomics data.

- It can be utilized in revealing the relationship between environmental factors and meta-omic samples directly through a nonlinear regression analysis.

- MetaComp is capable of automatically selecting the proper statistical method in two-group sample test thus improving experience for users that are not expertises of statistics.

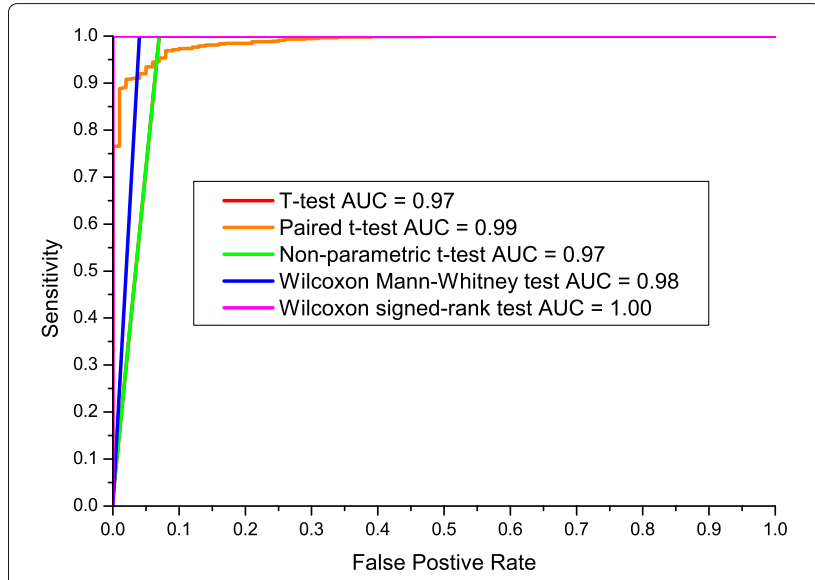

Fig. 7 ROC curve for all five methods. ROC performance of five methods in significant feature detection

MetaComp, as comprehensive analysis software for comparative meta-omics, takes advantages in incorporation of all types of meta-omics data, nonlinear regression analysis on environmental factors and automatical selection of suitable tests in two-group sample situation. These improvements meet the major demands in big data era of all types of meta-omics data. Moreover, according to our evaluation, MetaComp outperforms other methods by the automatically selected hypothesis testing method in detection of differentially abundant features. In brief, MetaComp is an integrative comparative meta-oimcs software designed for uncovering biological significant differences and providing visualization of these results for biologists. Moreover, it will throw light upon future comparative meta-omics studies on the complicated relationship between microbes and their living environments.

\section{Availability and requirements}

Project name: MetaComp.

Project home page: Homepage: http://cqb.pku.edu.cn/ ZhuLab/MetaComp/

GitHub page: https://github.com/pzhaipku/MetaComp/

Operating system(s): Linux and Windows 7, 8 and 10.

Programming language: $C \# \& R$

Other requirements: $\mathrm{R} 3.1 .3$ or higher.

Any restrictions to use by non-academics: none.

Table 9 Comparison of two-group sample test methods (FDR <0.05)

\begin{tabular}{|c|c|c|c|c|c|c|}
\hline Hypothesis test & True positive & False negative & False positive & True negative & Sensitivity & Specificity \\
\hline Non-parametric t-test & 65 & 35 & 0 & 900 & $65.0 \%$ & $100.0 \%$ \\
\hline Paired t-test & 100 & 0 & 386 & 524 & $100.0 \%$ & $57.6 \%$ \\
\hline t-test & 65 & 35 & 0 & 900 & $65.0 \%$ & $100.0 \%$ \\
\hline Wilcoxon rank-sum test & 44 & 56 & 0 & 900 & $44.0 \%$ & $100.0 \%$ \\
\hline Wilcoxon signed-rank test ${ }^{a}$ & 100 & 0 & 1 & 899 & $100.0 \%$ & $99.9 \%$ \\
\hline
\end{tabular}

aThis method is automatically selected by MetaComp 


\section{Additional files}

Additional file 1: Table S1. The input functional gene APM data of eight typical environmental metagenomic samples. (TXT $291 \mathrm{~kb}$ )

Additional file 2: Table S2. The detailed analysis results of eight typical environmental metagenomic samples. (XLS $2109 \mathrm{~kb}$ )

Additional file 3: Table S3. The input functional gene APM data of Acid Mine Drainage metaproteomic samples. (XLS 83kb)

Additional file 4: Table S4. The detailed analysis results of Acid Mine Drainage metaproteomic samples. (XLS $132 \mathrm{~kb}$ )

Additional file 5: Figure S1 to Figure S4. Visualized analysis results for Acid Mine Drainage metaproteomic samples and human fecal metabolomic samples. (PDF $204 \mathrm{~kb}$ )

Additional file 6: Table S5. The input metabolite APM data of human fecal metabolomic samples from healthy control people and NAFLD patients. (XLS 41 kb)

Additional file 7: Table S6. The detailed analysis results of human fecal metabolomic samples from healthy control people and NAFLD patients. (XLS $51 \mathrm{~kb})$

Additional file 8: Table S7. The input functional gene APM data of Hawaii Ocean metagenomic samples. (TXT 72kb)

Additional file 9: Table S8. The input vector of environmental factors related with Hawaii Ocean metagenomic samples. (TXT 1 kb)

Additional file 10: Table S9. The detailed analysis results of Hawaii Ocean metagenomic samples. (XLS $50 \mathrm{~kb}$ )

Additional file 11: Table S10. The input functional gene APM data of Acid Mine Drainage metagenomic samples. (TXT $62 \mathrm{~kb}$ )

Additional file 12: Table S11. The input vector of environmental factors related with Acid Mine Drainage metagenomic samples. (TXT $1 \mathrm{~kb}$ )

Additional file 13: Table S12. The detailed analysis results of Acid Mine Drainage metagenomic samples. (XLS $43 \mathrm{~kb}$ )

Additional file 14: Table S13. The original, resampled and downsampled datasets of twin gut data for comparison of significance detection. (XLS $622 \mathrm{~kb})$

Additional file 15: Table S14. The hypothesis testing results of t-test, paired t-test, non-parametric t-test, Wilcoxon rank-sum test and Wilcoxon signed-rank test. (XLS $168 \mathrm{~kb})$

\section{Abbreviations}

AMD: Acid mine drainage; amoA: Ammonia monooxygenase A; APM: Abundance profile matrix; ATP: Adenosine triphosphate; AUC: Area under ROC curve; DIP: Dissolved inorganic phosphate; DO: Dissolved oxygen; FDR: False discovery rate; fnr: Fumarate and nitrate reductase; FN: False negative; FP: False positive; IBS: Irritable bowel syndrome; LC: Liquid chromatography; MS: Mass spectrometry; NAFLD: Non-alcoholic fatty liver disease; OC: Oxygen content; OR: Odds ratio; OTU: Operational taxonomic units; PCA: Principal component analysis; ROC: Receiver operating characteristic curve; RPKM: Reads per kilobases million; SAM: Significance analysis of microarrays; SN: Sensitivity; SOTA: Self-organizing tree algorithm; SP: Specificity; TN: True negative; TOC: Total organic carbon; TP: True positive; VOC: Volate organic compounds

\section{Acknowledgements}

Not applicable.

\section{Funding}

This work was supported by the National Key Research and Development Program of China (2017YFC1200205), the National Natural Science Foundation of China (31671366 and 91231119), and the Special Research Project of "Clinical Medicine $+X$ " by Peking University. None of the funding bodies have played any part in the design of the study, in the collection, analysis, and interpretation of the data, or in the writing of the manuscript.

\section{Availability of data and materials}

The datasets supporting the conclusions of this article are downloadable at: http://cqb.pku.edu.cn/ZhuLab/MetaComp/download.html and available as additional files. The input data of eight typical environmental metagenomic samples were extracted from the published data of reference [57]. The input data of AMD metaproteomic samples were extracted from Table S5 of [10]. The input data of human fecal metabolomic samples were extracted from Additional file 1: Table S1 of [5]. The input data of Hawaii Ocean metagenomic samples were extracted from the published data of reference [7]. The input data of AMD metagenomic samples were extracted from the published data of reference [62]. The original data for the evaluation of two-group sample testing were extracted from the published data of reference [2].

\section{Authors' contributions}

PZ conceived and implemented the software, and wrote several sections of the manuscript. LY prepared datasets for MetaComp applications and wrote several sections. XG, ZW and XW applied MetaComp in meta-omics data listed in the manuscript. JG designed and developed a prototype of the software. $\mathrm{HZ}$ and LY critically revised the manuscript, provided valuable suggestions and supervised the whole work. All authors read and approved the final manuscript.

\section{Ethics approval and consent to participate}

Not applicable.

\section{Consent for publication}

Not applicable.

\section{Competing interests}

The authors declare that they have no competing interests.

\section{Publisher's Note}

Springer Nature remains neutral with regard to jurisdictional claims in published maps and institutional affiliations.

\section{Author details}

${ }^{1}$ State Key Laboratory for Turbulence and Complex Systems, Department of Biomedical Engineering, College of Engineering, Peking University, 100871 Beijing, China. ${ }^{2}$ Center for Quantitative Biology, Peking University, 100871 Beijing, China. ${ }^{3}$ Center for Protein Science, Peking University, 100871 Beijing, China.

Received: 18 July 2017 Accepted: 21 September 2017

Published online: 02 October 2017

\section{References}

1. White III RA, Callister SJ, Moore RJ, Baker ES, Jansson JK. The past, present and future of microbiome analyses. Nat Protoc. 2016;1 1(11):2049-53.

2. Turnbaugh PJ, Hamady M, Yatsunenko T, Cantarel BL, Duncan A, Ley RE, Sogin ML, Jones WJ, Roe BA, Affourtit JP, et al. A core gut microbiome in obese and lean twins. Nature. 2009:457(7228):480-4.

3. Wu GD, Chen J, Hoffmann C, Bittinger K, Chen YY, Keilbaugh SA, Sinha R. Linking long-term dietary patterns with gut microbial enterotypes. Science. 2011;334:105-8.

4. David LA, Maurice CF, Carmody RN, Gootenberg DB, Button JE, Wolfe BE, Biddinger SB. Diet rapidly and reproducibly alters the human gut microbiome. Nature. 2014;505:559-63.

5. Raman M, Ahmed I, Gillevet PM, Probert CS, Ratcliffe NM, Smith S, Greenwood R, Sikaroodi M, Lam V, Crotty P, et al. Fecal microbiome and volatile organic compound metabolome in obese humans with nonalcoholic fatty liver disease. Clin Gastroenterol Hepatol. 2013;11 (7): 868-75.

6. Liu Y, Zhang L, Wang X, Wang Z, Zhang J, Jiang R, Wang X, Wang K, Liu Z, Xia Z, et al. Similar fecal microbiota signatures in patients with diarrhea-predominant irritable bowel syndrome and patients with depression. Clin Gastroenterol Hepatol. 2016;14(11):1602-11.

7. DeLong EF, Preston CM, Mincer T, Rich V, Hallam SJ, Frigaard N. Community genomics among stratified microbial assemblages in the ocean's interior. Science. 2006;311:496-503.

8. Wang X, Wang Q, Guo X, Liu L, Guo J, Yao J, et al. Functional genomic analysis of hawaii marine metagenomes. Sci Bull. 2015;6:348-55.

9. Gilbert JA, Steele JA, Caporaso JG, Steinbrück L, Reeder J, Temperton B, Somerfield P. Defining seasonal marine microbial community dynamics. ISME J. 2012;6:298-308. 
10. Mueller RS, Dill BD, Pan C, Belnap CP, Thomas BC, VerBerkmoes NC, Hettich RL, Banfield JF. Proteome changes in the initial bacterial colonist during ecological succession in an acid mine drainage biofilm community. Environ Microbiol. 2011;13(8):2279-92.

11. Guo J, Wang Q, Wang X, Wang F, Yao J, Zhu H. Horizontal gene transfer in an acid mine drainage microbial community. BMC Genomics. 2015;16(1):496.

12. Sunagawa S, Coelho LP, Chaffron S, Kultima JR, Labadie K, Salazar G, Cornejo-Castillo FM. Structure and function of the global ocean microbiome. Science. 2015;348:1261359.

13. Rodriguez-Brito B, Rohwer F, Edwards RA. An application of statistics to comparative metagenomics. BMC Bioinforma. 2006;7:162.

14. Huson DH, Beier S, Flade I, Górska A, El-Hadidi M, Mitra S, Ruscheweyh H, Tappu R. Megan community edition-interactive exploration and analysis of large-scale microbiome sequencing data. PLoS Comput Biol. 2016;12(6):1004957.

15. Markowitz VM, Chen IA, Chu K, Szeto E, Palaniappan K, Pillay M, Ratner A, Huang J, Pagani I, Tringe S, et al. Img/m 4 version of the integrated metagenome comparative analysis system. Nucleic Acids Res. 2014;42(D1):568-73.

16. Parks DH, Tyson GW, Hugenholtz P, Beiko RG. Stamp: statistical analysis of taxonomic and functional profiles. Bioinformatics. 2014;30(21):3123-4.

17. Paulson JN, Pop M, Bravo HC. Metastats: an improved statistical method for analysis of metagenomic data. Genome Biol. 2011;12(1):1.

18. Sanli K, Karlsson FH, Nookaew I, Nielsen J. Fantom: Functional and taxonomic analysis of metagenomes. BMC Bioinforma. 2013;14:38.

19. Paulson JN, Stine OC, Bravo HC, Pop M. Differential abundance analysis for microbial marker-gene surveys. Nat Methods. 2013;10(12):1200-2.

20. Gowda H, Ivanisevic J, Johnson CH, Kurczy ME, Benton HP, Rinehart D, Nguyen T, Ray J, Kuehl J, Arevalo B, et al. Interactive xcms online: simplifying advanced metabolomic data processing and subsequent statistical analyses. Anal Chem. 2014;86(14):6931-9.

21. McDonald D, Clemente JC, Kuczynski J, Rideout JR, Stombaugh J, Wendel D, Wilke A, Huse S, Hufnagle J, Meyer F, et al. The biological observation matrix (biom) format or: how i learned to stop worrying and love the ome-ome. Gigascience. 2012;1(1):1.

22. Altschul SF, Gish W, Miller W, Myers EW, Lipman DJ. Basic local alignment search tool. J Mol Biol. 1990;215(3):403-10.

23. Mistry J, Finn RD, Eddy SR, Bateman A, Punta M. Challenges in homology search: Hmmer3 and convergent evolution of coiled-coil regions. Nucleic Acids Res. 2013;41(12):121-1.

24. Wood DE, Salzberg SL. Kraken: ultrafast metagenomic sequence classification using exact alignments. Genome Biol. 2014;15(3):1.

25. Glass EM, Wilkening J, Wilke A, Antonopoulos D, Meyer F. Using the metagenomics rast server (mg-rast) for analyzing shotgun metagenomes. Cold Spring Harb Protoc. 2010;2010(1):5368.

26. Pluskal T, Castillo S, Villar-Briones A, Orešič M. Mzmine 2: modular framework for processing, visualizing, and analyzing mass spectrometry-based molecular profile data. BMC Bioinforma. 2010;11(1):1

27. Brady A, Salzberg SL. Phymm and phymmbl: metagenomic phylogenetic classification with interpolated markov models. Nat Methods. 2009;6(9): 673-6.

28. Leimena MM, Ramiro-Garcia J, Davids M, van den Bogert B, Smidt H, Smid EJ, Boekhorst J, Zoetendal EG, Schaap PJ, Kleerebezem M. A comprehensive metatranscriptome analysis pipeline and its validation using human small intestine microbiota datasets. BMC Genomics. 2013;14(1):530.

29. Hettich RL, Sharma R, Chourey K, Giannone RJ. Microbial metaproteomics: identifying the repertoire of proteins that microorganisms use to compete and cooperate in complex environmental communities. Curr Opin Microbiol. 2012;15(3):373-80.

30. Wilmes P, Heintz-Buschart A, Bond PL. A decade of metaproteomics: Where we stand and what the future holds. Proteomics. 2015;15(20): 3409-17.

31. Wang Q, Garrity GM, Tiedje JM, Cole JR. Naive bayesian classifier for rapid assignment of rrna sequences into the new bacterial taxonomy Appl Environ Microbiol. 2007;73(16):5261-7.

32. Caporaso JG, Kuczynski J, Stombaugh J, Bittinger K, Bushman FD, Costello EK, Fierer N, Pena AG, Goodrich JK, Gordon Jl, et al. Qiime allows analysis of high-throughput community sequencing data. Nat Methods. 2010;7(5):335-6.
33. Schloss PD, Westcott SL, Ryabin T, Hall JR, Hartmann M, Hollister EB, Lesniewski RA, Oakley BB, Parks DH, Robinson CJ, et al. Introducing mothur: open-source, platform-independent, community-supported software for describing and comparing microbial communities. Appl Environ Microbiol. 2009;75(23):7537-41.

34. Cole JR, Wang Q, Fish JA, Chai B, McGarrell DM, Sun Y, Brown CT, Porras-Alfaro A, Kuske CR, Tiedje JM. Ribosomal Database Project: data and tools for high throughput rRNA analysis. Nucleic Acids Res. 2013;42(D1):D633-42.

35. DeSantis TZ, Hugenholtz P, Larsen N, Rojas M, Brodie EL, Keller K, Huber T, Dalevi D, Hu P, Andersen GL. Greengenes, a chimera-checked 16 s rna gene database and workbench compatible with arb. Appl Environ Microbiol. 2006;72(7):5069-72.

36. Quast C, Pruesse E, Yilmaz P, Gerken J, Schweer T, Yarza P, Peplies J, Glöckner FO. The silva ribosomal rna gene database project: improved data processing and web-based tools. Nucleic Acids Res. 2013;41(D1): 590-6.

37. Peng Y, Leung HC, Yiu SM, Chin FY. Idba-ud: a de novo assembler for single-cell and metagenomic sequencing data with highly uneven depth. Bioinformatics. 2012;28(11):1420-8.

38. Miller JR, Delcher AL, Koren S, Venter E, Walenz BP, Brownley A, Johnson J, Li K, Mobarry C, Sutton G. Aggressive assembly of pyrosequencing reads with mates. Bioinformatics. 2008;24(24):2818-24.

39. Lai B, Ding R, Li Y, Duan L, Zhu H. A de novo metagenomic assembly program for shotgun dna reads. Bioinformatics. 2012;28(11):1455-62.

40. Lai B, Wang F, Wang X, Duan L, Zhu H. Intemap: Integrated metagenomic assembly pipeline for ngs short reads. BMC Bioinforma. 2015;16(1):1.

41. Zhu W, Lomsadze A, Borodovsky M. Ab initio gene identification in metagenomic sequences. Nucleic Acids Res. 2010;38(12):132-2.

42. Kelley DR, Liu B, Delcher AL, Pop M, Salzberg SL. Gene prediction with glimmer for metagenomic sequences augmented by classification and clustering. Nucleic Acids Res. 2012;40(1):9-9.

43. Liu Y, Guo J, Hu G, Zhu H. Gene prediction in metagenomic fragments based on the svm algorithm. BMC Bioinforma. 2013;14:12.

44. Hu GQ, Guo JT, Liu YC, Zhu H. Metatisa: Metagenomic translation initiation site annotator for improving gene start prediction. Bioinformatics. 2009;25(14):1843-5.

45. Galperin MY, Makarova KS, Wolf YI, Koonin EV. Expanded microbial genome coverage and improved protein family annotation in the cog database. Nucleic Acids Res. 2015;43:261-9.

46. Kanehisa M, Sato Y, Kawashima M, Furumichi M, Tanabe M. Kegg as a reference resource for gene and protein annotation. Nucleic Acids Res. 2016;44(D1):D457-62.

47. Finn RD, Coggill P, Eberhardt RY, Eddy SR, Mistry J, Mitchell AL, Potter SC, Punta M, Qureshi M, Sangrador-Vegas A, et al. The pfam protein families database: towards a more sustainable future. Nucleic Acids Res. 2016;44(D1):279-85.

48. Overbeek R, Olson R, Pusch GD, Olsen GJ, Davis JJ, Disz T, Edwards RA, Gerdes S, Parrello B, Shukla M, et al. The seed and the rapid annotation of microbial genomes using subsystems technology (rast). Nucleic Acids Res. 2014;42(D1):206-14.

49. Grabherr MG, Haas BJ, Yassour M, Levin JZ, Thompson DA, Amit I, Adiconis X, Fan L, Raychowdhury R, Zeng Q, et al. Trinity: reconstructing a full-length transcriptome without a genome from rna-seq data. Nat Biotechnol. 2011;29(7):644.

50. Consortium $U$, et al. The universal protein resource (uniprot). Nucleic Acids Res. 2008;36(suppl 1):190-5.

51. Li H, Durbin R. Fast and accurate short read alignment with burrows-wheeler transform. Bioinformatics. 2009;25(14):1754-60

52. Langmead B, Salzberg SL. Fast gapped-read alignment with bowtie 2 . Nat Methods. 2012;9(4):357-9.

53. MacCoss MJ, Wu CC, Yates JR. Probability-based validation of protein identifications using a modified sequest algorithm. Anal Chem. 2002;74(21):5593-9.

54. Cottrell JS, London U. Probability-based protein identification by searching sequence databases using mass spectrometry data. Electrophoresis. 1999;20(18):3551-67.

55. Smith CA, O'Maille G, Want EJ, Qin C, Trauger SA, Brandon TR, Custodio DE, Abagyan R, Siuzdak G. Metlin: a metabolite mass spectral database. Ther Drug Monit. 2005;27(6):747-51. 
56. Horai H, Arita M, Kanaya S, Nihei Y, Ikeda T, Suwa K, Ojima Y, Tanaka K, Tanaka S, Aoshima K, et al. Massbank: a public repository for sharing mass spectral data for life sciences. J Mass Spectrom. 2010;45(7):703-14.

57. Tringe SG, von Mering C, Kobayashi A, Salamov AA, Chen K, Chang HW. Comparative metagenomics of microbial communities. Science. 2005;308:554-7.

58. Schumann W. Regulation of bacterial heat shock stimulons. Cell Stress Chaperones. 2016;21(6):959-68.

59. Nyström T. Stationary-phase physiology. Annu Rev Microbiol. 2004;58: 161-81.

60. Macnab RM. Genetics and biogenesis of bacterial flagella. Annu Rev Genet. 1992;26(1):131-58.

61. Nseir W, Nassar F, Assy N. Soft drinks consumption and nonalcoholic fatty liver. World J Gastroenterol. 2010;16(21):2579-88.

62. Kuang J, Huang L, He Z, Chen L, Hua Z, P J, et al. Predicting taxonomic and functional structure of microbial communities in acid mine drainage. ISME J. 2016;10:1527-39.

63. Jonsson V, Österlund T, Nerman O, Kristiansson E. Statistical evaluation of methods for identification of differentially abundant genes in comparative metagenomics. BMC Genomics. 2016;17(1):1.

\section{Submit your next manuscript to BioMed Central and we will help you at every step:}

- We accept pre-submission inquiries

- Our selector tool helps you to find the most relevant journal

- We provide round the clock customer support

- Convenient online submission

- Thorough peer review

- Inclusion in PubMed and all major indexing services

- Maximum visibility for your research

Submit your manuscript at www.biomedcentral.com/submit 